\title{
Analysis of Dynamic Stability of Nonlinear Suspension concerning Slowly Varying Sprung Mass
}

\author{
Jun Yao, Jinqiu Zhang, Mingmei Zhao, and Hu Peng \\ Brigade of Armament Trial and Training, Academy of Armored Force Engineering Institute, Beijing 100072, China \\ Correspondence should be addressed to Jun Yao; dryaojun@126.com
}

Received 20 November 2016; Revised 7 March 2017; Accepted 20 March 2017; Published 11 April 2017

Academic Editor: Dumitru I. Caruntu

Copyright (C) 2017 Jun Yao et al. This is an open access article distributed under the Creative Commons Attribution License, which permits unrestricted use, distribution, and reproduction in any medium, provided the original work is properly cited.

\begin{abstract}
In this paper, the stability of vehicle concerning the slow-varying sprung mass is analyzed based on two degrees of freedom quarter-car model. A mathematical model of vehicle is established, the nonlinear vibration caused by sprung mass vibration is solved, and frequency curve is obtained. The characteristics of a stable solution and the parameters affecting the stability are analyzed. The numeric solution shows that a slow-varying sprung mass is equivalent to adding a negative damping coefficient to the suspension system, making the effective damping coefficient change from negative to positive. Such changing parameters lead to Hopf bifurcation and a shrinking limit cycle. The simulation results indicate the existence of static as well as dynamic bifurcation and the result is a change in the final stable vibration of the suspension. Even the tiny vibration of the sprung mass will lead to amplitude mutation, leading to the sprung mass instability.
\end{abstract}

\section{Introduction}

The stability and bifurcation of a nonlinear system are closely related. Bifurcations that include both static and dynamic bifurcations affect the stability of the system. Static bifurcation such as saddle node bifurcation occurs mainly due to the nonlinear stiffness, discussed in vibration absorbers $[1,2]$. Dynamic bifurcation such as Hopf bifurcation occurs due to the parameter variations, reported in induction motor drive system [3] and circuit system [4]. They separated static bifurcation from dynamic bifurcation.

Research on static bifurcation varies. Static bifurcation caused by maneuvering load of an aircraft is analyzed in [5]. The bifurcation of a bearing system is studied in [6] and it was found that when the speed exceeds a critical value, the saddle node bifurcation occurs. The static bifurcation of an oscillator and its control is studied in $[7,8]$, illustrating the connection between nonlinear stiffness and saddle node bifurcation. Vibration analysis of a drill string using FEM [9] enables easy reconfiguration of the drill string for different boundary conditions. The model of these works is based on a smooth vibration state without any restriction. Compared with that, a vibro-impact system [10-13] is repeatedly reported. In these literatures, bifurcation of a piecewise-linear impact oscillator with drift is analyzed in [10]. Works in [11-13] concentrated on slip and contact motion of an oscillator excited by moving base. These researches go further to find more parameters which could result in bifurcation. However, the bifurcation is still static. The systems of all the works mentioned above can be treated as static models since their parameters remain still. But the parameters of any practical system always change with time.

Considering the case of high-speed rail CRH3 in China as the object, the critical speed leading to Hopf bifurcation between the wheel and the rail, which is critical for the safety of high-speed trains, is analyzed [14]. The stability of the rotational motion is studied in [15-17], of which the work presented in [16] analyzed an asymmetric rotation movement and found that both the Hopf bifurcation and the saddle node bifurcation will occur when the damping coefficient or the speed reaches a certain value. The kinematic stability of a symmetrical vane wheel of an aircraft is studied in [15] and it was found that any variation of the damping between the blade and the bearing, as well as the rotational speed, may lead to the generation of Hamilton Hopf bifurcation. Literatures [18-22] concern the effects of Coulomb damping on the 
system. Hopf bifurcation occurs as the effect of nonsmooth Coulomb friction [18]. After analyzing the vibration-impact dynamics of SDOF oscillator under the effect of Coulomb damping, it has been found out that there exist abundant dynamic phenomena, such as period-doubling routes to chaos $[19,20]$ and jump between multiperiodic orbits [21, 22]. A 1:2 resonant Hopf-Hopf bifurcation [23] and cellular shock instability are also studied concerning Hopf bifurcation [24]. Compared with the literature mentioned above, these references focused on the effect of parameter variations on the stability of the system. From these studies, it can be seen that the variations in system parameters can lead to the bifurcation. However, these studies are limited to the time delay and any changes in the characteristics of the system, such as variations in mass, stiffness, and damping coefficient, are neglected. The effect of the slowly varying parameters leading to bifurcation is rarely studied, and this kind of changes is widespread. Hence, it is worth paying attention to.

Slowly varying parameter defines the parameter which demands one-tenth time or less compared with other parameters with same self-variation in one system. Vehicle suspension is a system where the sprung mass is slowly varying. The fast-varying parameters like velocity or acceleration vary back and forth quickly. They are considered as variables in system equations. Different from that, the sprung mass is usually considered as constant. Actually, it decreases slowly, adding a slowly varying parameter to a suspension system, which changes the form of the equations. This is always ignored by researchers. For example, an adaptive control of nonlinear uncertain active suspension systems is analyzed in [25]. A vehicle seat with negative stiffness structures [26] and nonlinear asymmetrical shock absorber for vehicles [27] are presented. A controller considering actuator time delay is presented in $[28,29]$. They focus only on the control of nonlinear suspension and time delay, ignoring the slowly changing parameters, especially the variations in sprung mass. Enlighted by bifurcation research and the inadequate nonlinear suspension study mentioned above, we decide to figure out the effect of varying sprung mass on the stability of the nonlinear suspension.

This paper is organized as follows. In Section 2, a mathematical model for $1 / 4$ nonlinear suspension is established and is solved using the method of multiple scales. Section 3 analyzes the stability of the solution and possible bifurcation. Section 4 verifies the effectiveness of the proposed theoretical analysis. Section 5 concludes the paper.

\section{Governing Equation for Nonlinear Suspension with Slowly Varying Sprung Mass under Forced Vibration}

Leaving the turn of the vehicle out of account, the suspension model can be described in Figure 1, which includes eccentric mass $m_{e}$, the sprung mass $m_{s}(\tau)$, and the unsprung mass $m_{u}$, connected by a nonlinear spring $k_{s}\left(\delta_{s}\right)$ and a shock absorber $c_{s}\left(\xi_{s}\right)$. Since the sprung mass is a slowly varying parameter, it is $m_{s}(\tau)$ instead of $m_{s}(t)$ based on the definition of slowly varying parameter mentioned in Introduction. The

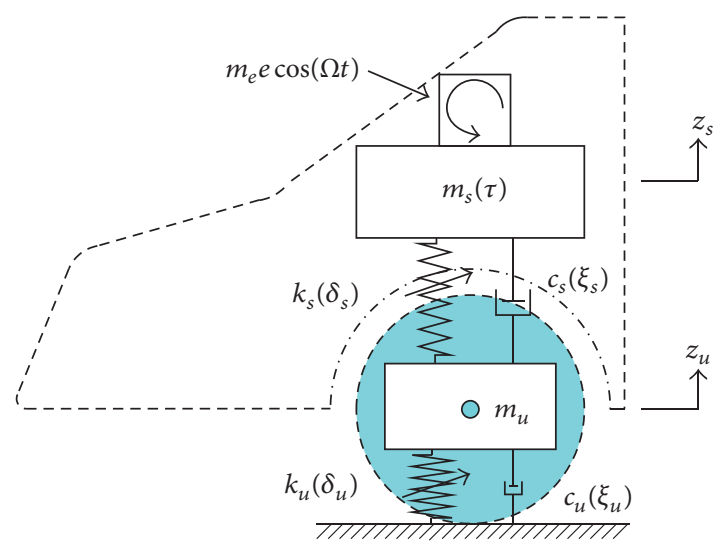

FIGURE 1: Nonlinear quarter-car model.

wheel can be simplified as a nonlinear spring $k_{u}\left(\delta_{u}\right)$ and a damper $c_{u}\left(\xi_{u}\right)$. When the automobile travels in the freeway, the construction vehicle crawls in the construction site, or the military vehicle slows down preparing for shooting, the unevenness of the road is small, which can be treated as a plane. And the vertical vibration is $m_{e} e \cos (\Omega t)$.

According to Newton's second law, the governing equation is

$$
\begin{aligned}
& \frac{d}{d t}\left[m_{s}(\tau) \frac{d z_{s}}{d t}\right]+f_{c s}\left(\frac{d z_{s}}{d t}-\frac{d z_{u}}{d t}\right) \\
& \quad+f_{k s}\left(z_{s}-z_{u}\right)=m_{e} e \cos (\Omega t), \\
& m_{u} \frac{d^{2} z_{s}}{d t^{2}}-f_{c s}\left(\frac{d z_{s}}{d t}-\frac{d z_{u}}{d t}\right)-f_{k s}\left(z_{s}-z_{u}\right) \\
& \quad+f_{c u}\left(\frac{d z_{u}}{d t}\right)+f_{k u}\left(z_{u}\right)=0,
\end{aligned}
$$

where $f_{c s}\left(d z_{s} / d t-d z_{u} / d t\right)=c_{s}\left(d z_{s} / d t-d z_{u} / d t\right)+$ $\xi_{s}\left(d z_{s} / d t-d z_{u} / d t\right)^{3}, f_{k s}\left(z_{s}-z_{u}\right)=k_{s}\left(z_{s}-z_{u}\right)+\delta_{s}\left(z_{s}-\right.$ $\left.z_{u}\right)^{3}, f_{c u}\left(d z_{s} / d t\right)=c_{u}\left(d z_{s} / d t\right)+\xi_{u}\left(d z_{s} / d t\right)^{3}, f_{k u}\left(z_{u}\right)=$ $k_{u} z_{u}+\delta_{u} z_{u}{ }^{3}$. The damping term and the spring term share the same form $a x+b x^{3}$ in accordance with literatures $[29,30]$.

Owing to the fact that the slow time satisfies $\tau=\varepsilon t$, so we can get $d / d t=(d / d \tau)(d \tau / d t)=\varepsilon(d / d \tau)$. And (1a) and (1b) can be simplified as follows:

$$
\begin{gathered}
m_{s}(\tau) \ddot{z}_{s}+\varepsilon m^{\prime}(\tau) \dot{z}_{s}+c_{s}\left(\dot{z}_{s}-\dot{z}_{u}\right)+\xi_{s}\left(\dot{z}_{s}-\dot{z}_{u}\right)^{3} \\
+k_{s}\left(z_{s}-z_{u}\right)+\delta_{s}\left(z_{s}-z_{u}\right)^{3}=m_{e} e \cos (\Omega t), \\
m_{u} \ddot{z}_{u}-c_{s}\left(\dot{z}_{s}-\dot{z}_{u}\right)-\xi_{s}\left(\dot{z}_{s}-\dot{z}_{u}\right)^{3}-k_{s}\left(z_{s}-z_{u}\right) \\
-\delta_{s}\left(z_{s}-z_{u}\right)^{3}+c_{u} \dot{z}_{u}+\xi_{u} \dot{z}_{u}{ }^{3}+k_{u} z_{u}+\delta_{u} z_{u}^{3}=0,
\end{gathered}
$$

where $m^{\prime}(\tau)=d m(\tau) / d \tau, \ddot{z}_{s}=d^{2} z_{s} / d t^{2}, \dot{z}_{s}=d z_{s} / d t, \ddot{z}_{u}=$ $d^{2} z_{u} / d t^{2}, \dot{z}_{u}=d z_{u} / d t$. 
Comparing to suspension system with constant sprung mass, this system contains an additional damping term $\varepsilon m^{\prime}(\tau) \dot{z}_{s}$, and the damping coefficient $\varepsilon m^{\prime}(\tau)$ of this term is the function of the slowly varying sprung mass. When the sprung mass varies from big to small, this slowly varying damping coefficient is negative, resulting in selfexcited vibration. Meanwhile, the natural frequency is slowly varying, leading to slowly varying amplitude and phase angle.

The sprung mass vibration makes the whole suspension in a forced vibration state. Equations (2a) and (2b) can be changed as

$$
\begin{aligned}
\ddot{x}+ & \varepsilon \mu \dot{x}+2 \varsigma_{x} w_{x}(\dot{x}-\dot{y})+2 \zeta_{x} w_{x}(\dot{x}-\dot{y})^{3} \\
& +w_{x}^{2}(x-y)+\beta_{x}(x-y)^{3}=f \cos (\Omega t), \\
\ddot{y}- & 2 \varsigma_{x y} w_{x y}(\dot{x}-\dot{y})-2 \zeta_{x y} w_{x y}(\dot{x}-\dot{y})^{3}-w_{x y}{ }^{2} x \\
& -\beta_{x y}(x-y)^{3}+2 \varsigma_{y} w_{y} \dot{y}+w_{y}^{2} y+\beta_{y} y^{3}=0,
\end{aligned}
$$

where $z_{s}=x, z_{u}=y, \mu=m_{s}{ }^{\prime}(\tau) / m_{s}(\tau), \varsigma_{x}=c_{s} / 2 w_{x} m_{s}(\tau)$, $\zeta_{x}=\xi_{s} / 2 w_{x} m_{s}(\tau), w_{x}^{2}=k_{s} / m_{s}(\tau), \beta_{x}=\delta_{s} / m_{s}(\tau), \varsigma_{x y}=$ $c_{s} / 2 w_{x y} m_{u}, \zeta_{x y}=\xi_{s} / 2 w_{x y} m_{u}, w_{x y}^{2}=k_{s} / m_{u}, \beta_{x y}=\delta_{s} / m_{u}$, $\varsigma_{y}=c_{u} / 2 w_{y} m_{u}, w_{y}{ }^{2}=\left(k_{s}+k_{u}\right) / m_{u}, \beta_{y}=\delta_{u} / m_{u}, f=m_{e} e /$ $m_{s}(\tau)$.

The sprung mass varies due to the change of load. When $m_{s} \approx 9 m_{u} k_{s} /\left(k_{s}+k_{u}\right)$, the $3: 1$ internal resonance occurs.

Because the exact solution of (3a) and (3b) cannot be found, this paper applies the method of multiple scales to solve the equation. A small perturbation parameter $\varepsilon$ is introduced, and the scale transformation is carried out.

$$
\begin{gathered}
\varsigma_{x} \longrightarrow \varepsilon \varsigma_{x}, \\
\zeta_{x} \longrightarrow \varepsilon \zeta_{x}, \\
\varsigma_{x y} \longrightarrow \varepsilon \varsigma_{x y}, \\
\zeta_{x y} \longrightarrow \varepsilon \zeta_{x y}, \\
\beta_{x} \longrightarrow \varepsilon \beta_{x}, \\
\beta_{x y} \longrightarrow \varepsilon \beta_{x y}, \\
w_{x y}{ }^{2} x \rightarrow \varepsilon w_{x y}{ }^{2} x, \\
f \longrightarrow \varepsilon f, \\
\varsigma_{y} \longrightarrow \varepsilon^{2} \varsigma_{y}, \\
\beta_{y} \longrightarrow \varepsilon^{2} \beta_{y} .
\end{gathered}
$$

Because the stiffness of the tire $k_{u}$ is much bigger, the nonlinearity term $\delta_{u}$ is much smaller than that of the spring, and the equivalent damping coefficient $c_{u}$ is much smaller than the damper, the perturbation parameter is $\varepsilon^{2}(4 \mathrm{~b})$ when rescaling stiffness and damping coefficient of the tire in the same equation.
Substituting (4a) and (4b) into (3a) and (3b) and retaining $\varepsilon^{0}$ and $\varepsilon^{1}$ terms yield the following equations:

$$
\begin{aligned}
\ddot{x} & \varepsilon \mu \dot{x}+2 \varepsilon \zeta_{x} w_{x}(\dot{x}-\dot{y})+2 \varepsilon \zeta_{x} w_{x}(\dot{x}-\dot{y})^{3} \\
& +w_{x}^{2}(x-y)+\varepsilon \beta_{x}(x-y)^{3}=\varepsilon f \cos (\Omega t), \\
\ddot{y} & -2 \varepsilon \zeta_{x y} w_{x y}(\dot{x}-\dot{y})-2 \varepsilon \zeta_{x y} w_{x y}(\dot{x}-\dot{y})^{3}-\varepsilon w_{x y}{ }^{2} x \\
& -\varepsilon \beta_{x y}(x-y)^{3}+w_{y}^{2} y=0 .
\end{aligned}
$$

The term $w_{x y}{ }^{2} x$ in (3b) is much smaller in value than the term $w_{y}^{2} y$, as such this term can be considered as a small perturbation term. Because of that, it can be rescaled as $\varepsilon w_{x y}{ }^{2} x$ in (5b).

According to the method of multiscale, the approximate solution can be expressed as

$$
\begin{aligned}
& x(t, \varepsilon)=x_{0}\left(T_{0}, T_{1}\right)+\varepsilon x_{1}\left(T_{0}, T_{1}\right)+O\left(\varepsilon^{2}\right), \\
& y(t, \varepsilon)=y_{0}\left(T_{0}, T_{1}\right)+\varepsilon y_{1}\left(T_{0}, T_{1}\right)+O\left(\varepsilon^{2}\right),
\end{aligned}
$$

where $T_{0}=t$ can be seen as faster time scale and $T_{1}=\varepsilon t$ can be seen as slower time scale.

Under 3:1 internal resonance condition,

$$
\begin{gathered}
\Omega=w_{x}+\varepsilon \sigma, \\
w_{y}=3 w_{x}+\varepsilon \sigma_{2},
\end{gathered}
$$

where $\sigma$ is a detuning parameter expressing the closeness of $\Omega$ to $w_{x}$ and $\sigma_{2}$ is a detuning parameter expressing the closeness of $w_{y}$ to $3 w_{x}$.

There are differential operators like these

$$
\begin{aligned}
\frac{d}{d t}(\cdot) & =\left(D_{0}+\varepsilon D_{1}\right)(\cdot), \\
\frac{d^{2}}{d t^{2}}(\cdot) & =\left(D_{0}^{2}+2 \varepsilon D_{0} D_{1}\right)(\cdot), \\
D_{0} & =\frac{\partial}{\partial T_{0}} \\
D_{1} & =\frac{\partial}{\partial T_{1}}
\end{aligned}
$$

Substituting (6a), (6b), (7a), (7b), (8a), and (8b) into (5a) and (5b) and listing equations with the same order of $\varepsilon$ result in the following equations:

$$
\begin{gathered}
\varepsilon^{0}: D_{0}^{2} x_{0}+w_{x}^{2} x_{0}=w_{y}^{2} y_{0}, \\
D_{0}^{2} y_{0}+w_{y}^{2} y_{0}=0
\end{gathered}
$$




$$
\begin{aligned}
\varepsilon^{1}: D_{0}^{2} x_{1}+w_{x}^{2} x_{1}= & -2 D_{0} D_{1} x_{0}-\mu D_{0} x_{0} \\
& -2 \varsigma_{x} w_{x} D_{0}\left(x_{0}-y_{0}\right) \\
& -2 \zeta_{x} w_{x}\left[D_{0}\left(x_{0}-y_{0}\right)\right]^{3} \\
& +w_{x}^{2} y_{1}-\beta_{x}\left(x_{0}-y_{0}\right)^{3} \\
& +f \cos \left(\Omega T_{0}\right), \\
D_{0}^{2} y_{1}+w_{y}^{2} y_{1}= & -2 D_{0} D_{1} y_{0} \\
& +2 \varsigma_{x y} w_{x y} D_{0}\left(x_{0}-y_{0}\right) \\
& +2 \zeta_{x y} w_{x y}\left[D_{0}\left(x_{0}-y_{0}\right)\right]^{3} \\
& +w_{x y}^{2} x_{0}+\beta_{x y}\left(x_{0}-y_{0}\right)^{3} .
\end{aligned}
$$

The general solution of (9a) and (9b) can be expressed as

$$
\begin{aligned}
& x_{0}=G B e^{i w_{y} T_{0}}+G \bar{B} e^{-i w_{y} T_{0}}+A e^{i w_{x} T_{0}}+\bar{A} e^{-i w_{x} T_{0}}, \\
& y_{0}=B e^{i w_{y} T_{0}}+\bar{B} e^{-i w_{y} T_{0}},
\end{aligned}
$$

where amplitudes $A$ and $B$ are functions of slower time $T_{1}$. $\bar{A}$ and $\bar{B}$ are the complex conjugates of $A$ and $B$ and $G=$ $w_{x}^{2} /\left(w_{x}^{2}-w_{y}^{2}\right)$.

Substituting (11a) and (11b) into (10a) and (10b) and eliminating the secular terms result in the following equations:

$$
\begin{aligned}
& -2 A^{\prime} i w_{x}-\mu A i w_{x}-2 \varsigma_{x} w_{x} A i w_{x} \\
& -2 \varsigma_{x} w_{x} i\left[3 A^{2} \bar{A} w_{x}^{3}+6 A B \bar{B}(G-1)^{2} w_{x} w_{y}^{2}\right. \\
& \left.+3 \bar{A}^{2} B(1-G) w_{x}^{2} w_{y} e^{i \sigma_{2} T_{1}}\right]-\beta_{x}\left[3 A^{2} \bar{A}\right. \\
& \left.+6 A B \bar{B}(G-1)^{2}+3 \bar{A}^{2} B(G-1) e^{i \sigma_{2} T_{1}}\right]+\frac{1}{2} \\
& +f e^{i \sigma T_{1}}=0 \\
& -2 B^{\prime} i w_{y}-2 \varsigma_{x y} w_{x y} B i w_{y}+2 \varsigma_{x y} w_{x y} G B i w_{y} \\
& +2 \zeta_{x y} w_{x y} i\left[6 A \bar{A} B(G-1) w_{x}^{2} w_{y}\right. \\
& \left.+3 B^{2} \bar{B}(G-1)^{3} w_{y}^{3}-A^{3} w_{x}^{3} e^{-i \sigma_{2} T_{1}}\right]+w_{x y}^{2} G B \\
& +\beta_{x y}\left[6 A \bar{A} B(G-1)+3 B^{2} \bar{B}(G-1)^{3}\right. \\
& \left.+A^{3} e^{-i \sigma_{2} T_{1}}\right]=0 .
\end{aligned}
$$

Change $A$ and $B$ into polar form:

$$
\begin{aligned}
& A=\frac{1}{2} a e^{i \beta}, \\
& B=\frac{1}{2} b e^{i \theta},
\end{aligned}
$$

where $a, b, \beta$, and $\theta$ are real functions of slower time $T_{1}$.
Substituting (13a) and (13b) into (12a) and (12b) and separating real and imaginary parts yield the following equations:

$$
\begin{aligned}
a^{\prime}= & -l_{1} a-h_{1} a^{3}-h_{2} a b^{2}-h_{3} a^{2} b \cos (\phi-3 \gamma) \\
& +h_{4} a^{2} b \sin (\phi-3 \gamma)-h_{5} \sin \gamma, \\
a \gamma^{\prime}= & -g_{1} a+h_{6} a^{3}+h_{7} a b^{2}-h_{3} a^{2} b \sin (\phi-3 \gamma) \\
& -h_{4} a^{2} b \cos (\phi-3 \gamma)-h_{5} \cos \gamma, \\
b^{\prime}= & -l_{2} b-s_{1} a^{2} b-s_{2} b^{3}-s_{3} a^{3} \cos (\phi-3 \gamma) \\
& -s_{4} a^{3} \sin (\phi-3 \gamma), \\
b \phi^{\prime}= & -g_{2} b+s_{5} a^{2} b+s_{6} b^{3}+s_{3} a^{3} \sin (\phi-3 \gamma) \\
& -s_{4} a^{3} \cos (\phi-3 \gamma),
\end{aligned}
$$

where $l_{1}=\mu / 2+w_{x} \varsigma_{x}, h_{1}=(3 / 4) \zeta_{x} w_{x}^{3}, h_{2}=(3 /$ 2) $\zeta_{x} w_{x} w_{y}^{2}(G-1)^{2}, h_{3}=(3 / 4) \zeta_{x} w_{x}^{2} w_{y}(1-G), h_{4}=-(3 / 8)\left(\beta_{x} /\right.$ $\left.w_{x}\right)(G-1), h_{5}=(1 / 2)\left(f / w_{x}\right), g_{1}=\sigma, h_{6}=(3 / 8)\left(\beta_{x} / w_{x}\right)$, $h_{7}=(3 / 4)\left(\beta_{x} / w_{x}\right)(G-1)^{2}, l_{2}=\varsigma_{x y} w_{x y}(1-G), s_{1}=-(3 /$ 2) $\zeta_{x y} w_{x y} w_{x}^{2}(G-1), s_{2}=-(3 / 4) \zeta_{x y} w_{x y} w_{y}^{2}(G-1)^{3}, s_{3}=(1 /$ 4) $\zeta_{x y} w_{x y}\left(w_{x}^{3} / w_{y}\right), s_{4}=-(1 / 8)\left(\beta_{x y} / w_{y}\right), g_{2}=3 \sigma-\sigma_{2}+(1 /$ 2) $\left(w_{x y}{ }^{2} / w_{y}\right) G, s_{5}=-(3 / 4)\left(\beta_{x y} / w_{y}\right)(G-1), s_{6}=-\left(3 \beta_{x y} /\right.$ $\left.8 w_{y}\right)(G-1)^{3}, \gamma=\beta-\sigma T_{1}, \phi=\theta+\sigma_{2} T_{1}-3 \sigma T_{1}$.

\section{Stability Analysis of Solutions}

3.1. Frequency Response and Stable Region of Solution. Since sprung mass $m(\tau)$ is function of slow time scale $\tau$, it can be considered constant in fast time scale $t$. The steady-state response of system can be sought by letting $a^{\prime}=b^{\prime}=0$ and $\gamma^{\prime}=\phi^{\prime}=0$ in (14a) and (14b). There are two kinds of solutions, uncoupled case $a \neq 0, b=0$ and coupled case $a \neq 0, b \neq 0$. As for uncoupled case, (14a) and (14b) can be simplified into

$$
h_{5}^{2}-\left(l_{1} a+h_{1} a^{3}\right)^{2}-\left(g_{1} a-h_{6} a^{3}\right)^{2}=0
$$

The number of solutions concerning (15) can be 1 or 3 . When the intensity of excitation from sprung mass vibration is beyond a certain value, the number of solutions is 3, resulting in saddle node bifurcation and jump phenomenon in amplitude-frequency curve. When the intensity is within this value, only one solution can be sought. And this critical value can be solved when there is only one solution in (15) throughout the whole frequency band.

$$
\begin{aligned}
& s_{5 \_ \text {stable }} \\
& \quad=\sqrt{\frac{8}{27\left(h_{1}{ }^{2}+h_{6}{ }^{2}\right)^{2}}\left(l_{1} h_{6} \frac{h_{1}+\sqrt{3} h_{6}}{h_{6}-\sqrt{3} h_{1}}-l_{1} h_{1}\right)^{3}} .
\end{aligned}
$$


As can be seen from (16), this critical value is related to damping coefficient and nonlinearity stiffness of the spring.

Combining (7a), (7b), (11a), and (11b) and (13a) and (13b), then we get

$$
\begin{aligned}
& x=G b \cos (3 \Omega t+\phi)+a \cos (\Omega t+\gamma)+O(\varepsilon), \\
& y=b \cos (3 \Omega t+\phi)+O(\varepsilon) .
\end{aligned}
$$

From (17a) and (17b) we can see that, in the coupled case, there exist two vibration frequencies, that is, forced vibration frequency $\Omega$ and free vibration frequency $3 \Omega$, due to the existence of internal resonance. The nonlinear stiffness of the spring makes the free vibration frequency exactly three times the forcing frequency. As for uncoupled case, the vehicle vibration is mainly reflected by the vibration of sprung mass, and there is only one vibration frequency in the vehicle.

When the suspension is in the uncoupled case, the steady statement of sprung mass vibration is that of autonomous system in the singularity $(\bar{a}, \bar{r})$. Linearize the system.

Linearizing (14a) and (14b) in the singularity $(\bar{a}, \bar{r})$ yields the following equation about disturbance of $\Delta \bar{a}$ and $\Delta \bar{r}$ :

$$
\begin{aligned}
& \frac{d \Delta \bar{a}}{d T_{1}}=-l_{1} \Delta \bar{a}-3 h_{1} \bar{a}^{2} \Delta \bar{a}-h_{5} \cos \bar{\gamma} \Delta \bar{\gamma}, \\
& \frac{d \Delta \bar{\gamma}}{d T_{1}}=2 h_{6} \bar{a} \Delta \bar{a}+\frac{h_{5}}{\bar{a}^{2}} \cos \bar{\gamma} \Delta \bar{a}+\frac{h_{5}}{\bar{a}} \sin \bar{\gamma} \Delta \bar{\gamma} .
\end{aligned}
$$

Use $a^{\prime}=\gamma^{\prime}=0$ to eliminate $\bar{\gamma}$ in the former equation and secular equation can be got:

$$
\operatorname{det}\left[\begin{array}{cc}
-l_{1}-3 h_{1} \bar{a}^{2}-\lambda & N \\
M & -l_{1}-h_{1} \bar{a}^{2}-\lambda
\end{array}\right]=0,
$$

where $M=(1 / \bar{a})\left[-g_{1}+3 h_{6} \bar{a}^{2}\right], \quad N=-\bar{a}\left[-g_{1}+h_{6} \bar{a}^{2}\right]$.

Expand (19):

$$
\begin{aligned}
\lambda^{2}+ & \left(2 l_{1}+4 h_{1} \bar{a}^{2}\right) \lambda+l_{1}{ }^{2}+4 l_{1} h_{1} \bar{a}^{2}+3 h_{1}{ }^{2} \bar{a}^{4}+g_{1}{ }^{2} \\
& -4 h_{6} g_{1} \bar{a}^{2}+3 h_{6}{ }^{2} \bar{a}^{4}=0 .
\end{aligned}
$$

As for $2 l_{1}+4 h_{1} \bar{a}^{2}>0$, the condition of losing stability for steady-state solution is

$$
l_{1}^{2}+g_{1}^{2}-4\left(l_{1} h_{1}-h_{6} g_{1}\right) \bar{a}^{2}+3\left(h_{1}^{2}+h_{6}^{2}\right) \bar{a}^{4}<0 .
$$

The stable region is solved from (21).

3.2. Stability of Solutions Accompanied with Bifurcation. In the slow time scale $\tau$, the excitation only changes the position of the equilibrium point of the dynamic bifurcation. It does not change the nature of the bifurcation. Therefore, the excitation is out of consideration when analyzing the dynamic bifurcation of the suspension. We can make $h_{5}=0$. As for uncoupled case, (14a) and (14b) can be transformed into

$$
\begin{aligned}
& a^{\prime}=-l_{1} a-h_{1} a^{3}, \\
& \gamma^{\prime}=-g_{1}+h_{6} a^{2} .
\end{aligned}
$$

Focus on (22a) and let

$$
z(a) \stackrel{\text { def }}{=}-l_{1} a-h_{1} a^{3}=0 .
$$
is,

Then we get two solution curves intersecting at $(0,0)$. That

$$
\begin{aligned}
& a=0, \\
& a=\sqrt{-\frac{l_{1}}{h_{1}} .}
\end{aligned}
$$

They are point and circle in polar coordinates, which correspond to the equilibrium point and limit cycle of the two-dimensional systems (22a) and (22b). Focus on its stability and define

$$
z^{\prime}(a)=\frac{d z}{d a}=-l_{1}-3 h_{1} a^{2} .
$$

For trivial solution $a=0: z^{\prime}(0)=-l_{1}$. At the time $l_{1}>0$, $a=0$ is asymptotic stable. At the time $l_{1}<0,(0,0)$ is not stable.

For nontrivial solution $a=\sqrt{-l_{1} / h_{1}}: z^{\prime}\left(\sqrt{-l_{1} / h_{1}}\right)=2 l_{1}$. At the time $l_{1}<0$, the system has asymptotically stable solution. And at the time $l_{1}>0$, the system has unstable solutions. The former corresponds to a supercritical pitchfork bifurcation, while the latter corresponds to subcritical pitchfork bifurcation.

For the nonlinear suspension system, the sprung mass $m(\tau)$ reduced slowly from large to small. In the beginning when $l_{1}<0$, the system has nontrivial solution $a=\sqrt{-l_{1} / h_{1}}$. As the sprung mass continues decreasing, the limit cycle is reduced until the trivial solution $a=0$ emerges. Then, the sprung mass continues to decrease, $l_{1}>0$, and the system solution is asymptotically stable. In the range $l_{1}<0$, the initial amplitude inside the limit cycle increases and that outside the limit cycle reduces, all converging in the stable limit cycle in the end. This process leads to change in the static bifurcation nature of some initial values after they go through the dynamic bifurcation, destroying the system stability.

\section{Numerical Validation}

4.1. Frequency Response and Stable Region of Solution. Let $m_{u}=45 \mathrm{~kg}, k_{s}=2000 \mathrm{~N} / \mathrm{m}, \delta_{s}=660 \mathrm{~N} / \mathrm{m}, k_{u}=$ $16000 \mathrm{~N} / \mathrm{m}$. When the damper is out of work, the damping decreases sharply to $c_{s}=20 \mathrm{Ns} / \mathrm{m}, \xi_{s}=0.3 \mathrm{Ns} / \mathrm{m}$. At the same time, the slowly varying sprung mass satisfies

$$
m_{s}(\tau)= \begin{cases}80 e^{-(5 / 9) \tau} & \tau \leq 0.52 \\ 45 & \tau>0.52\end{cases}
$$

When $\tau>0.52$, the sprung mass remains constant, and $w_{y}=$ $20 \mathrm{rad} / \mathrm{s}, w_{x}=6.6667 \mathrm{rad} / \mathrm{s}$, which means $w_{y} \approx 3 w_{x}$. These parameters are simplified from real trucks like SY5500THB, SY5411THB, and XZJ5470THB53. 


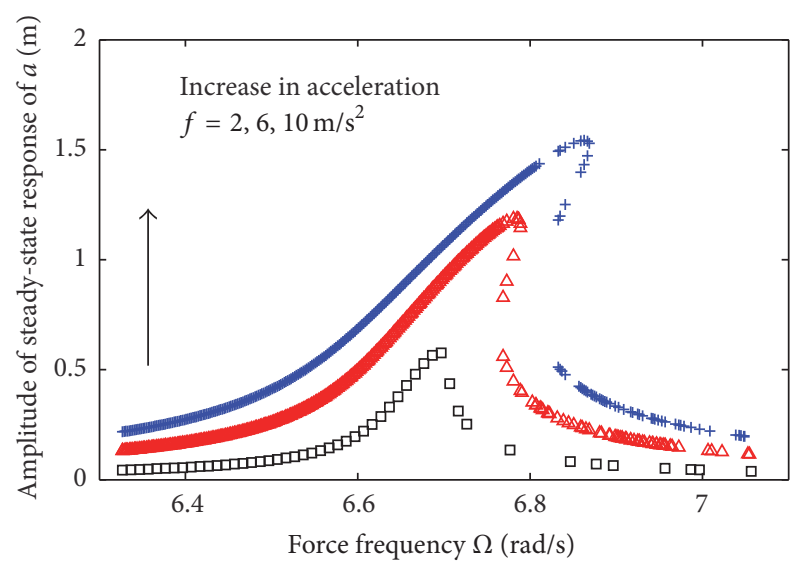

FIGURE 2: The effect of varying acceleration $f$ on frequency response of vibration $a$. $f=2 \mathrm{~m} / \mathrm{s}^{2}$ (black squares) and $f=6 \mathrm{~m} / \mathrm{s}^{2}$ (red triangles), $f=10 \mathrm{~m} / \mathrm{s}^{2}$ (blue crosses).

According to (16), the maximum excitation acceleration in which the bifurcation does not occur in the steady-state is $f_{\text {stable }}=2 w_{x} s_{5 \text { stable }}=2.8677 \mathrm{~m} / \mathrm{s}^{2}$. When the excitation acceleration increases, the amplitude of the sprung mass vibration will show bifurcation caused by the saddle node, that is, jumping phenomenon in the amplitude-frequency curve.

Figure 2 shows that when the excitation acceleration is less than $f_{\text {stable, }}$, the amplitude-frequency curve is singlevalued and remains the same in the forward frequency sweeping or backward frequency sweeping. When the excitation acceleration is much bigger than $f_{\text {stable }}$, jump phenomenon occurs. When the frequency is swept up, the amplitude is getting higher to the top and then jumps down to a much lower level. As the frequency continues to increase, the amplitude declines. When the frequency is swept down, the opposite jump-up phenomenon will occur. In other words, there exists jump phenomenon caused by bifurcation in amplitude-frequency curve. The curve is different in the forward frequency sweeping or backward frequency sweeping. With the increase of excitation acceleration, the vibration amplitude of the sprung mass under all frequency bands increases, and the unstable frequency band increases.

Figures 3 and 4 are frequency response curves under the conditions of $\delta_{s}=660 \mathrm{~N} / \mathrm{m}$ and $\xi_{s}=0.3 \mathrm{Ns} / \mathrm{m}$, respectively, and $f_{\text {stable }}=2.8677 \mathrm{~m} / \mathrm{s}^{2}$ in common. As can be seen in Figure 3, when the nonlinear damping coefficient of the damper is bigger than $0.3 \mathrm{Ns} / \mathrm{m}$, under which the solution is stable, the steady-state solution of the sprung mass vibration does not bifurcate. On the contrary, bifurcation occurs in the steady-state solution of the sprung mass vibration. And the smaller the damping coefficient is, the wider the unstable bands become. Different from Figure 2, only the amplitude of which the frequency is close to the resonant frequency will get larger as the nonlinear damping coefficient decreases. Those beyond the resonant frequency change later. This indicates that the decreasing nonlinear damping coefficient

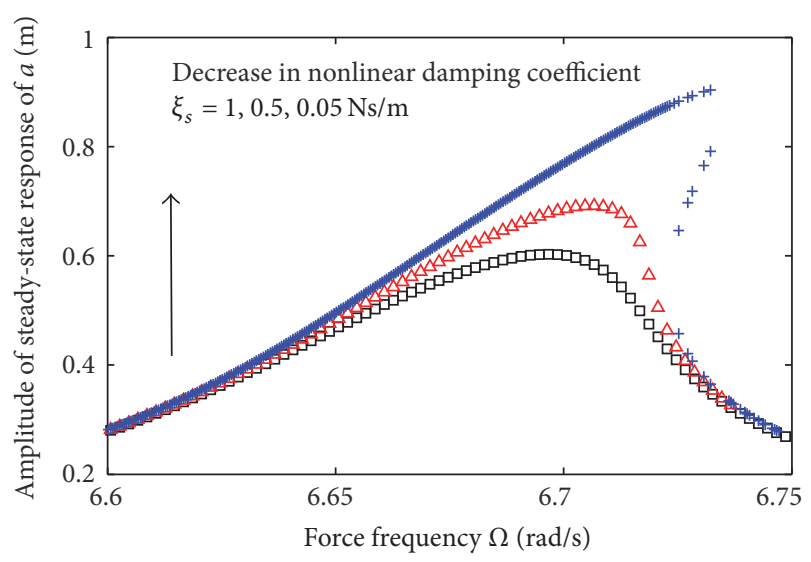

FIGURE 3: The effect of varying nonlinear damping coefficient $\xi_{s}$ on frequency response of vibration $a . \xi_{s}=1 \mathrm{Ns} / \mathrm{m}$ (black squares), $\xi_{s}=$ $0.5 \mathrm{Ns} / \mathrm{m}$ (red triangles), and $\xi_{s}=0.05 \mathrm{Ns} / \mathrm{m}$ (blue crosses).



FIGURE 4: The effect of varying nonlinear stiffness $\delta_{s}$ on frequency response of vibration $a . \delta_{s}=60 \mathrm{~N} / \mathrm{m}$ (black squares), $\delta_{s}=460 \mathrm{~N} / \mathrm{m}$ (red triangles), and $\delta_{s}=860 \mathrm{~N} / \mathrm{m}$ (blue crosses).

has effect on the amplitude of which the frequency is close to the resonant frequency, enlarging both the amplitude and the unstable frequency bands. In the rest of the frequency band, the amplitude has nothing to do with the damping coefficient. Figure 4 shows that the nonlinearity of the spring does not increase the vibration amplitude of the sprung mass but causes the resonance point to shift to right and generates unstable bands. Concluding from Figures 2, 3, and 4 , the increasing acceleration of the excitation, the decreasing nonlinear damping coefficient of the damper, and the increasing nonlinearity of the spring result in wider and wider unstable frequency bands.

From Figure 2, it can be found that when the amplitudefrequency curve shows an unstable frequency band, the excitation acceleration and force frequency will show multiple values. This is reflected more clearly in Figure 5. Let $\xi_{s}=$ $0.3 \mathrm{Ns} / \mathrm{m}$ and $\delta_{s}=660 \mathrm{~N} / \mathrm{m}$; then we get Figure 5. In a smaller force frequency like $\Omega=6.67 \mathrm{rad} / \mathrm{s}$, the amplitude of the sprung mass vibration is a single-valued curve; when 


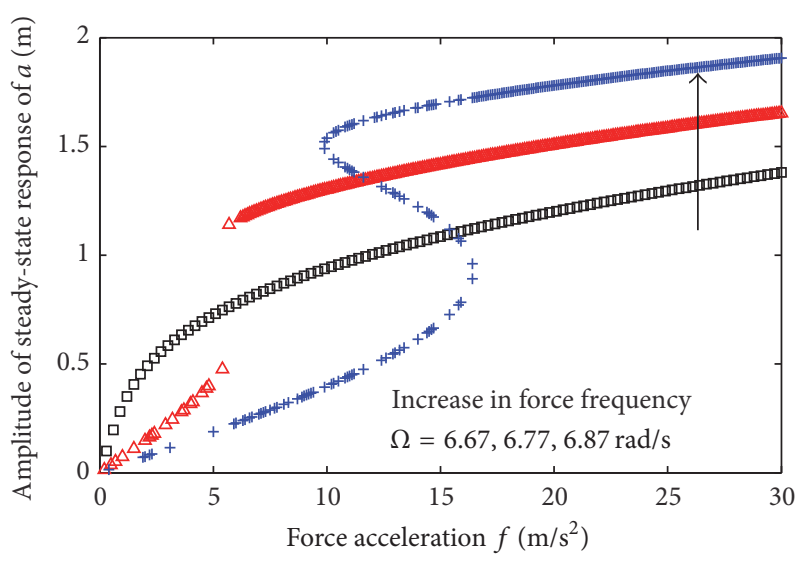

FIGURE 5: The effect of varying force frequency $\Omega$ on force acceleration response of vibration $a . \Omega=6.67 \mathrm{rad} / \mathrm{s}$ (black squares), $\Omega=6.77 \mathrm{rad} / \mathrm{s}$ (red triangles), and $\Omega=6.87 \mathrm{rad} / \mathrm{s}$ (blue crosses).

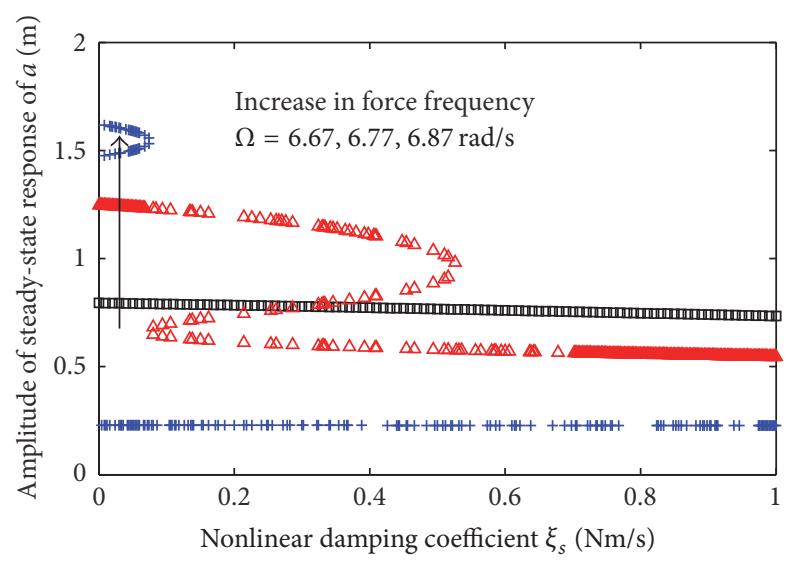

FIGURE 6: The effect of varying force frequency $\Omega$ on nonlinear damping coefficient response of vibration $a . \Omega=6.67 \mathrm{rad} / \mathrm{s}$ (black squares), $\Omega=6.77 \mathrm{rad} / \mathrm{s}$ (red triangles), and $\Omega=6.87 \mathrm{rad} / \mathrm{s}$ (blue crosses).

$\Omega=6.77 \mathrm{rad} / \mathrm{s}$ and $\Omega=6.87 \mathrm{rad} / \mathrm{s}$, the curve changes to multivalue. Similar to Figure 2, in the multivalue curve, there is a jumping phenomenon in the process of increasing or decreasing the excitation acceleration and the acceleration response curve is different. The force frequency gets bigger; the unstable interval of the excitation acceleration gets wider and gradually shifts to right. Different from Figure 2, with small excitation acceleration, the amplitude decreases as the force frequency increases; with big excitation acceleration, the opposite will occur.

Figures 6 and 7 are response curves under the conditions of $\delta_{s}=660 \mathrm{~N} / \mathrm{m}$ and $\xi_{s}=0.3 \mathrm{Ns} / \mathrm{m}$, respectively, and $f_{\text {stable }}=$ $6 \mathrm{~m} / \mathrm{s}^{2}$ in common. As can be seen in Figure 6, in a small force frequency like $\Omega=6.67 \mathrm{rad} / \mathrm{s}$, the amplitude of the sprung mass vibration is a single-valued curve, which decreases as the damping coefficient increases. When $\Omega=6.77 \mathrm{rad} / \mathrm{s}$ and $\Omega=6.87 \mathrm{rad} / \mathrm{s}$, the curve changes to multivalue. In the process of increasing in the nonlinear damping coefficient,

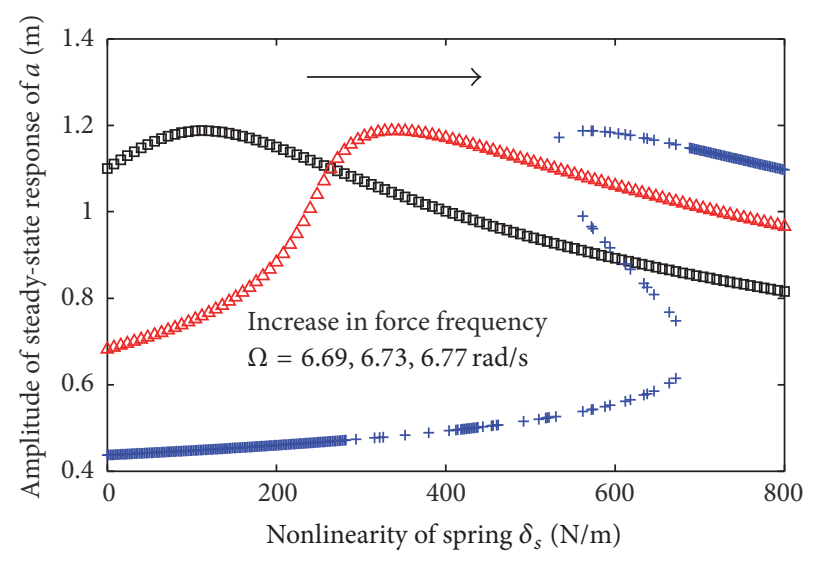

FIGURE 7: The effect of varying force frequency $\Omega$ on nonlinear stiffness response of vibration $a . \Omega=6.69 \mathrm{rad} / \mathrm{s}$ (black squares), $\Omega=6.73 \mathrm{rad} / \mathrm{s}$ (red triangles), and $\Omega=6.77 \mathrm{rad} / \mathrm{s}$ (blue crosses).

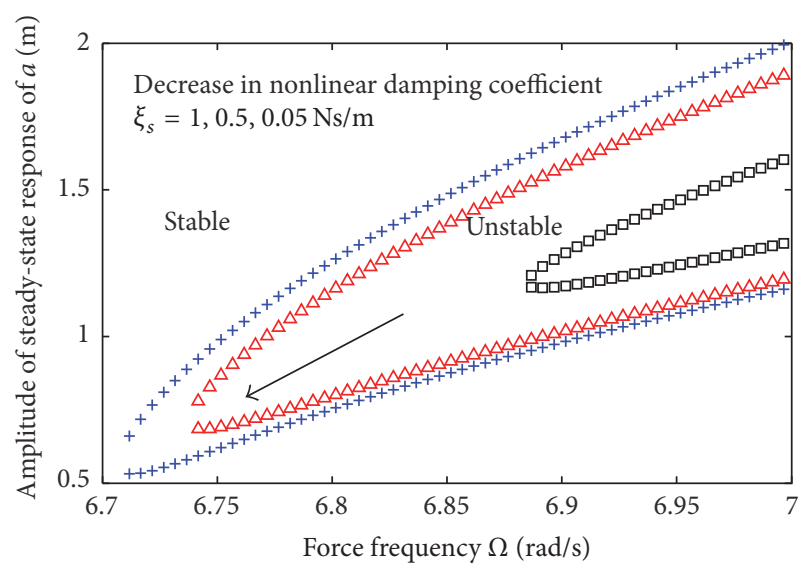

FIGURE 8: The effect of varying nonlinear damping coefficient $\xi_{s}$ on unstable area of vibration $a . \xi_{s}=1 \mathrm{Ns} / \mathrm{m}$ (black squares), $\xi_{s}=$ $0.5 \mathrm{Ns} / \mathrm{m}$ (red triangles), and $\xi_{s}=0.05 \mathrm{Ns} / \mathrm{m}$ (blue crosses).

the unstable damping coefficient interval under small force frequency gradually moves to right and decreases, and curves converge in the end. Figure 7 shows that when the nonlinear stiffness of the spring increases, the bigger the force frequency is, the larger the vibration amplitude is, and the unstable nonlinear stiffness interval increases and shifts to right. But the maximum amplitude of the sprung mass vibration in the whole nonlinear stiffness range is equal.

Let the nonlinear stiffness $\delta_{s}=660 \mathrm{~N} / \mathrm{m}$ and the nonlinear damping coefficient $\xi_{s}=0.3 \mathrm{Ns} / \mathrm{m}$; Figures 8 and 9 are obtained. As can be seen in Figure 8, with the increase of the damping coefficient, the unstable region reduces along the direction of the saddle node. Similar to Figure 8, as the nonlinear stiffness increases, the whole unstable region in Figure 10 enlarges and gradually moves down.

Let the nonlinear damping coefficient $\xi_{s}=0.3 \mathrm{Ns} / \mathrm{m}$ and the nonlinear stiffness $\delta_{s}=660 \mathrm{~N} / \mathrm{m}$; Figures 10 and 11 are obtained. Both Figures 10 and 11 indicate that, in the whole range of nonlinear damping coefficient, the force frequency 


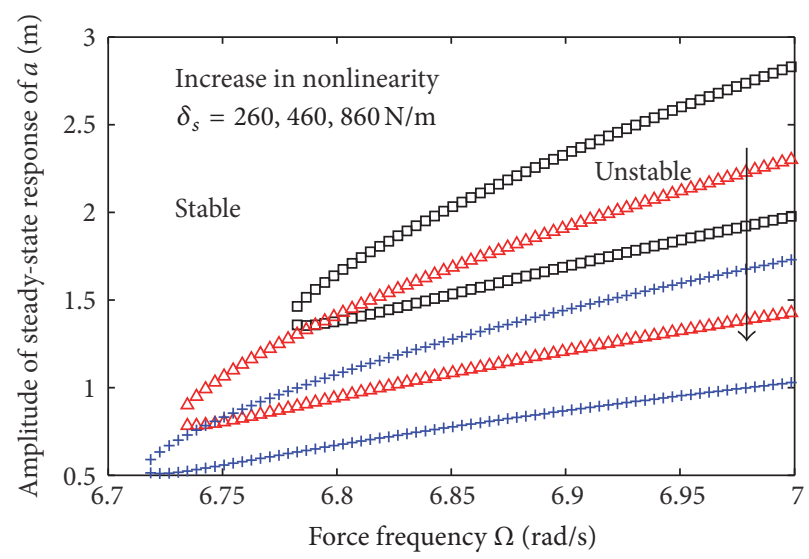

FIGURE 9: The effect of varying nonlinear stiffness $\delta_{s}$ on unstable area of vibration $a . \delta_{s}=260 \mathrm{~N} / \mathrm{m}$ (black squares), $\delta_{s}=460 \mathrm{~N} / \mathrm{m}$ (red triangles), and $\delta_{s}=860 \mathrm{~N} / \mathrm{m}$ (blue crosses).



Figure 10: The effect of varying force frequency $\Omega$ on unstable area of vibration $a . \Omega=6.77 \mathrm{rad} / \mathrm{s}$ (black squares), $\Omega=6.87 \mathrm{rad} / \mathrm{s}$ ( $\mathrm{red}$ triangles), and $\Omega=6.97 \mathrm{rad} / \mathrm{s}$ (blue crosses).

increases, and the unstable region increases and moves to upper right.

\subsection{Stability of Solutions Accompanied with Bifurcation.} When $\tau>0.52$, the sprung mass remains constant and the saddle node bifurcation occurs.

The critical lower limit frequency is $w_{\text {down }}$; the critical upper limit frequency is $w_{\text {upper }}$. When the force frequency $\Omega$ gets close to $w_{\text {down }}$, all vibrations under different initial conditions are attracted to the stable focus $P_{1}$. Once the force frequency $\Omega$ satisfies $\Omega>w_{\text {down }}$, another stable focus $P_{3}$ is generated, which is shown in Figures 12(a) and 12(b). It can be seen in Figure 12(c), when the force frequency $\Omega$ satisfies $w_{\text {down }}<\Omega<w_{\text {upper }}$, there exist two stable focuses $P_{1}, P_{3}$ and a saddle node $P_{2}$. Only certain initial leads to trajectory which can get to the saddle node $P_{2}$. With a small disturbance, the system state will be attracted to focus $P_{1}$ or $P_{3}$. The dashed line in Figure 12(c) is the dividing line of the two regions. The state outside the dashed line is attracted to the stable focus $P_{3}$ and those inside the dashed line is attracted to the stable focus $P_{1}$. As the force frequency continues to increase, at the time $\Omega>w_{\text {upper }}$, the focus $P_{1}$ disappears, and all states are attracted to the focus $P_{3}$. The region covered by red dash dotted line varies from large to small and ultimately disappears. These phenomena indicate that the upper and the lower two solutions are asymptotically stable in the amplitude-frequency curve, and the intermediate solution is unstable. Because of the fact that only asymptotically stable motion can be achieved in actual physical world, the jumping phenomenon can be watched.

When $\tau \leq 0.52$, the sprung mass changes slowly, and dynamic Hopf bifurcation occurs. The limit cycle shrinks as the sprung mass reduces. The initial amplitude inside the limit cycle increases and that outside the limit cycle reduces, all converging in the stable limit cycle in the end. At that time $\tau=0.52$, due to the impact of Hopf bifurcation, different initials converge in the limit cycle $a=0.7$, as shown in Figure 13.

This process changes the initial value. Since the nonlinear vibration is closely related to initial state, it will change the nature of the vibration.

In Figure 14, blue line represents vibration beginning with the initial value $(2,0.7)$ while the red line represents vibration beginning with the initial value $(0.7,0.7)$. The initial $(0.7$, 


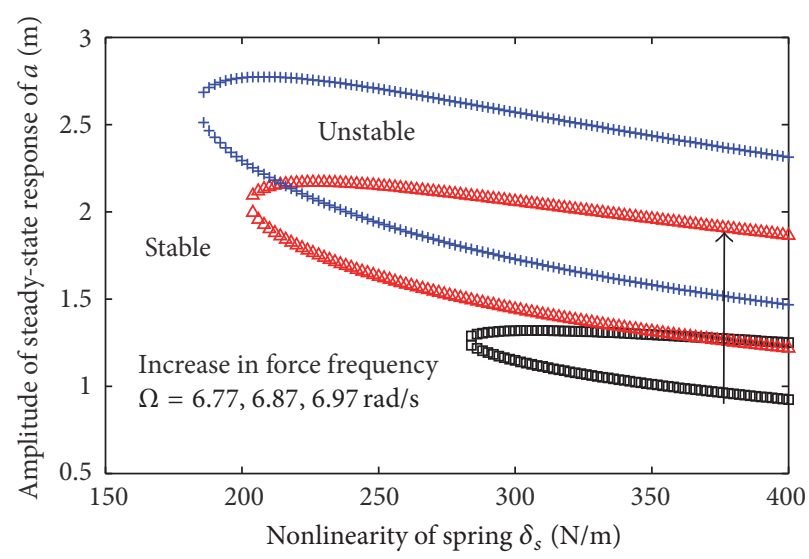

FIGURE 11: The effect of varying force frequency $\Omega$ on unstable area of vibration $a . \Omega=6.72 \mathrm{rad} / \mathrm{s}$ (black squares), $\Omega=6.77 \mathrm{rad} / \mathrm{s}$ (red triangles), and $\Omega=6.82 \mathrm{rad} / \mathrm{s}$ (blue crosses).

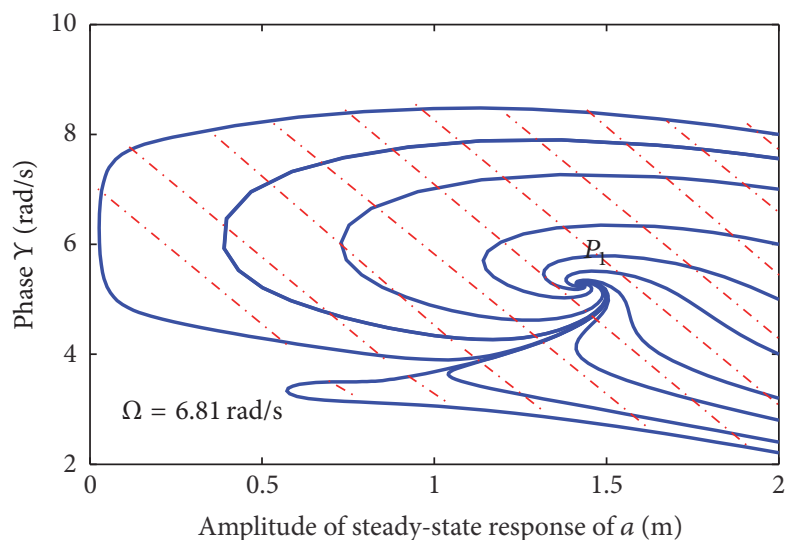

(a)



(c)

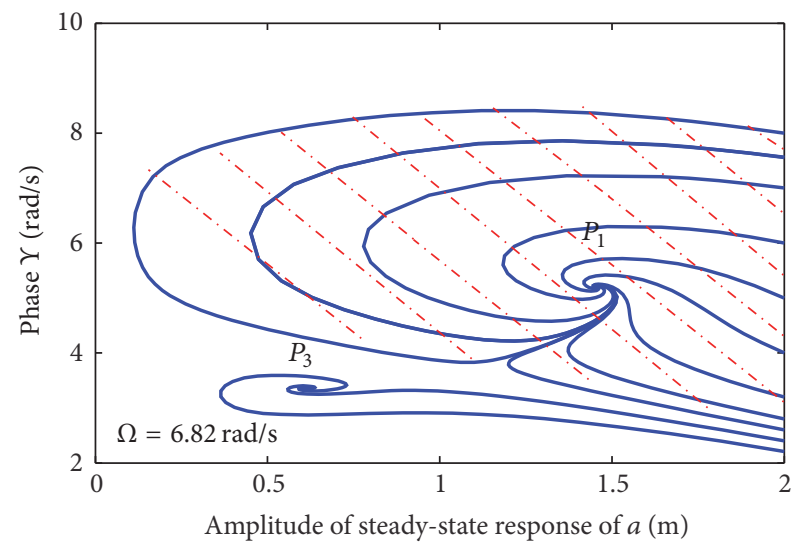

(b)

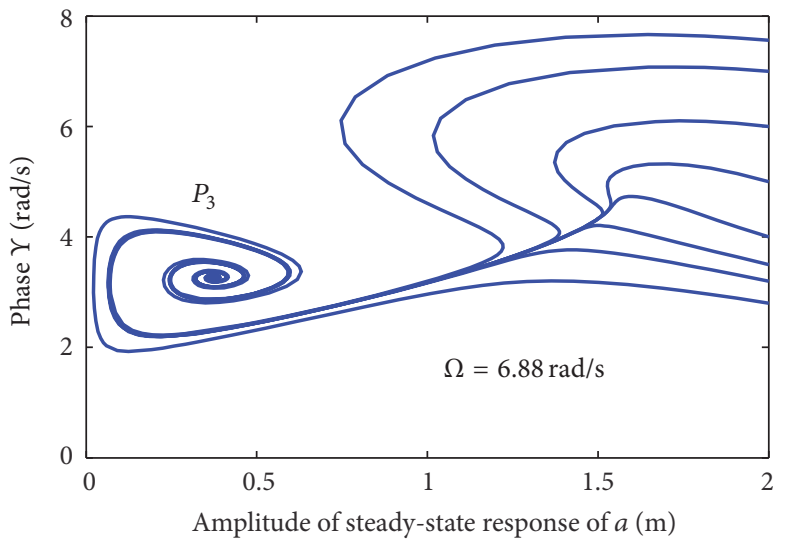

(d)

FIGURE 12: Evolution of the phase plane with the force frequency: (a) 6.81, (b) 6.82, (c) 6.85, and (d) $6.88 \mathrm{rad} / \mathrm{s}$.

$0.7)$ can be obtained from initial $(2,0.7)$ after being affected by Hopf bifurcation. As can be seen in Figure 14(b), when the excitation frequency satisfies $w_{\text {down }}<\Omega<w_{\text {upper }}$, the final stable state is different from these two initial values. At the time $\tau=0.52$, the effect of the Hopf bifurcation changes the initial value of the blue line to the initial value of the red line. Once the sprung mass is constant, the Hopf bifurcation disappears and the new initial value is affected by the saddle node bifurcation. These indicate that the stability of the system is extremely fragile. 


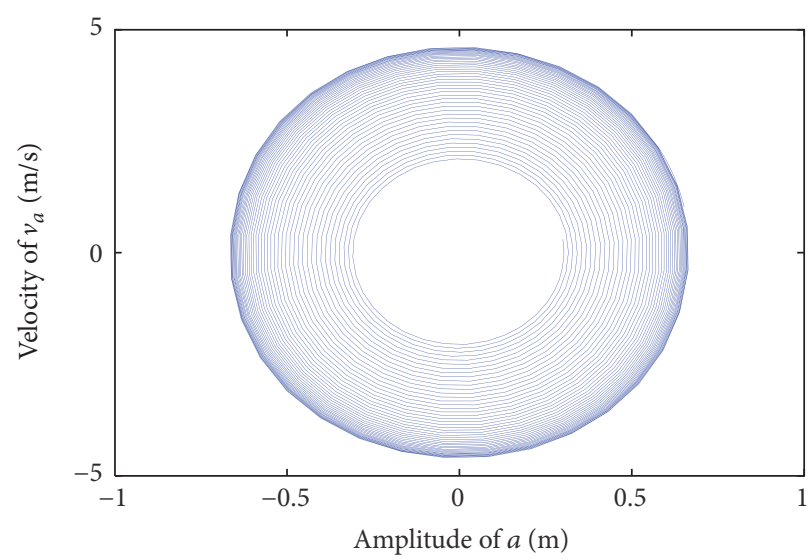

(a)



(c)



(b)

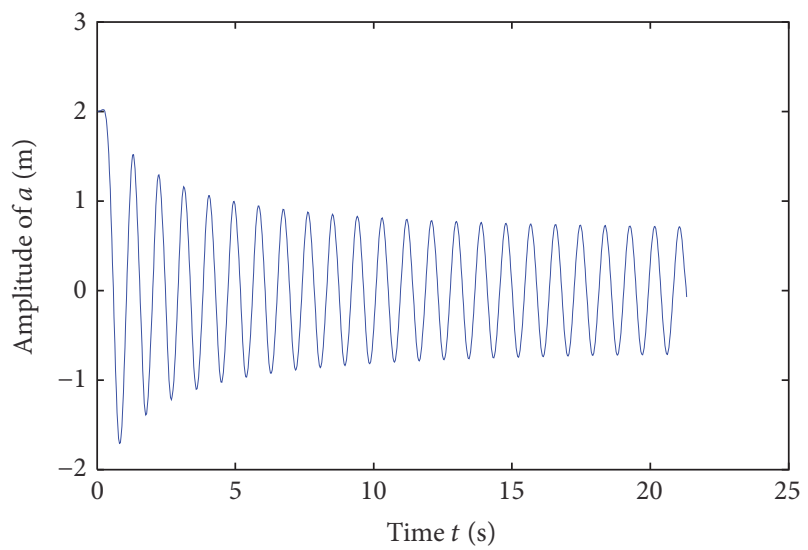

(d)

FIgURE 13: Phase portrait and time-domain vibration amplitude of Hopf bifurcation.

\section{Conclusion}

In this paper, the stability of vehicle concerning the slowvarying sprung mass is analyzed based on two-degree-offreedom quarter-car model. The vibration amplitude solution is solved using multiscale method, followed by numerical validation. After analyzing amplitude-frequency curve, phase plane of steady-state response and phase portrait, and timedomain vibration amplitude of Hopf bifurcation, the main points of our work can be concluded as follows:

(a) There exist rich mechanical phenomena, which include internal resonance, jumping phenomenon, and Hopf bifurcation.

When the unsprung mass $m_{u}$, sprung mass $m_{s}$, the linear stiffness of tire $k_{u}$, and the linear stiffness of the suspension spring $k_{s}$ satisfy the condition $m_{s} \approx 9 m_{u} k_{s} /\left(k_{s}+k_{u}\right)$, the vibration frequency ratio of unsprung mass and sprung mass is $3: 1$, and internal resonance will occur. Under the condition of internal resonance, when the sprung mass and the unsprung mass are not coupled, the vibration amplitude of the sprung mass creates a jumping phenomenon. Meanwhile, since the sprung mass is slowly varying, dynamic Hopf bifurcation occurs. (b) The essential of the effect of slowly varying sprung mass is changing the damping coefficient.

The numeric solution shows that a slow-varying sprung mass is equivalent to adding a negative damping coefficient to the suspension system, making the effective damping coefficient change from negative to positive. Such changing parameters lead to Hopf bifurcation and a shrinking limit cycle.

(c) The stability of nonlinear suspension is closely related to damping coefficient, nonlinear stiffness, and a slowly varying sprung mass.

Because of the slowly varying process, vibration with different initial states converges in the stable limit cycle in the end. They cannot result in final vibration with different states. Since the nonlinear vibrations are closely related to the initial states, Hopf bifurcation can change the final stable state of the vibration.

All of the above observations indicate the existence of static as well as dynamic bifurcation and the result is a change in the final stable vibration of the suspension. Even the tiny vibration of the sprung mass will lead to amplitude mutation, leading to the sprung mass instability. 




(a)

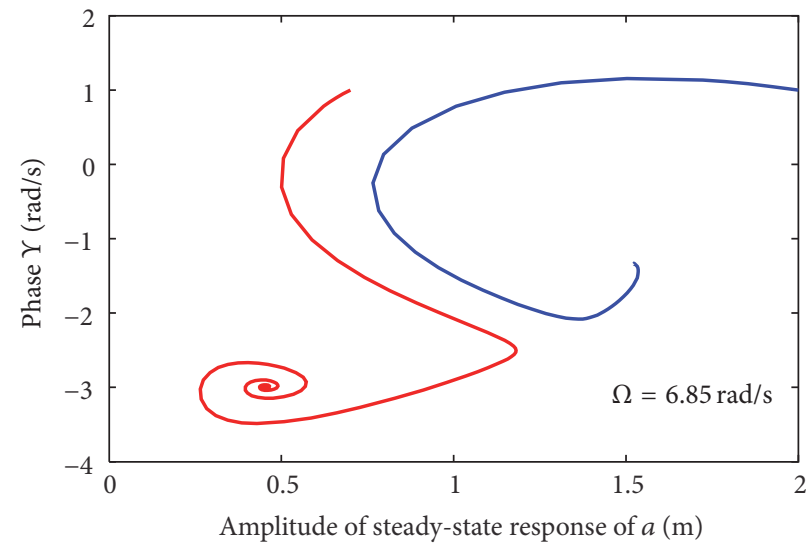

(b)

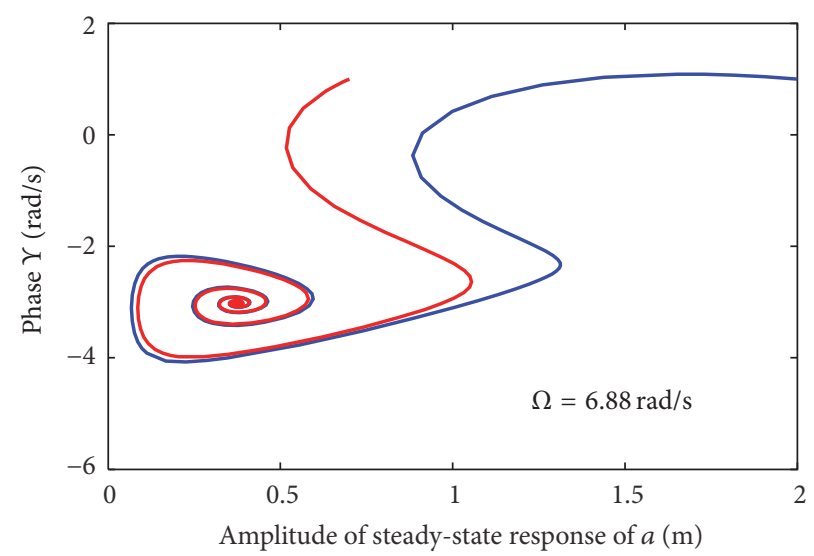

(c)

FIgURE 14: Different solution induced by Hopf bifurcation.

\section{Conflicts of Interest}

The authors declare that no conflicts of interest exist regarding the publication of this paper.

\section{References}

[1] M. Febbo and S. P. MacHado, "Nonlinear dynamic vibration absorbers with a saturation," Journal of Sound and Vibration, vol. 332, no. 6, pp. 1465-1483, 2013.

[2] F. Djemal, F. Chaari, J.-L. Dion, F. Renaud, I. Tawfiq, and M. Haddar, "Performance of a non linear dynamic vibration absorbers," Journal of Mechanics, vol. 31, no. 3, pp. 345-353, 2015.

[3] P. S. Deivasundari, G. Uma, and R. Santhi, "Experimental verification of Hopf bifurcation in pulse-width modulated inverter fed cage induction motor drive system," IET Power Electronics, vol. 7, no. 2, pp. 340-349, 2014.

[4] X. J. Han, F. B. Xia, P. Ji, Q. S. Bi, and J. Kurths, "Hopfbifurcation-delay-induced bursting patterns in a modified circuit system," Communications in Nonlinear Science and Numerical Simulation, vol. 36, pp. 517-527, 2016.

[5] L. Hou, Y. S. Chen, Z. Y. Lu, and Z. G. Li, "Bifurcation analysis for 2:1 and 3:1 super-harmonic resonances of an aircraft cracked rotor system due to maneuver load," Nonlinear Dynamics, vol. 81, no. 1-2, pp. 531-547, 2015.

[6] N. A. Saeed, M. Eissa, and W. A. El-Ganini, "Nonlinear oscillations of rotor active magnetic bearings system," Nonlinear Dynamics, vol. 74, no. 1-2, pp. 1-20, 2013.

[7] M. A. Acar and C. Yilmaz, "Design of an adaptive-passive dynamic vibration absorber composed of a string-mass system equipped with negative stiffness tension adjusting mechanism," Journal of Sound and Vibration, vol. 332, no. 2, pp. 231-245, 2013.

[8] A. M. Elnaggar and K. M. Khalil, "Control of the nonlinear oscillator bifurcation under superharmonic resonance," Journal of Applied Mechanics and Technical Physics, vol. 54, pp. 34-43, 2013.

[9] A. Ghasemloonia, D. G. Rideout, and S. D. Butt, "Vibration analysis of a drillstring in vibration-assisted rotary drilling: finite element modeling with analytical validation," Journal of Energy Resources Technology, vol. 135, no. 3, Article ID 032902, 18 pages, 2013.

[10] J. P. Chávez, E. Pavlovskaia, and M. Wiercigroch, "Bifurcation analysis of a piecewise-linear impact oscillator with drift," Nonlinear Dynamics, vol. 77, no. 1-2, pp. 213-227, 2014.

[11] U. Andreaus and N. Nisticò, "An analytical-numerical model for contact-impact problems: theory and implementation in a 
two-dimensional distinct element algorithm," Computer Modeling and Simulation in Engineering, vol. 3, no. 2, pp. 98-110, 1998.

[12] U. Andreaus and P. Casini, "Friction oscillator excited by moving base and colliding with a rigid or deformable obstacle," International Journal of Non-Linear Mechanics, vol. 37, no. 1, pp. 117-133, 2002.

[13] U. Andreaus and P. Casini, "Dynamics of friction oscillators excited by a moving base and/or driving force," Journal of Sound and Vibration, vol. 245, no. 4, pp. 685-699, 2001.

[14] T. T. Zhang and H. Y. Dai, "Bifurcation analysis of high-speed railway wheel-set," Nonlinear Dynamics, vol. 83, no. 3, pp. 15111528, 2016.

[15] A. Najafi, M.-R. Ghazavi, and A.-A. Jafari, "Stability and Hamiltonian HOPf bifurcation for a nonlinear symmetric bladed rotor," Nonlinear Dynamics, vol. 78, no. 2, pp. 1049-1064, 2014.

[16] M. Shahgholi and S. E. Khadem, "Hopf bifurcation analysis of asymmetrical rotating shafts," Nonlinear Dynamics, vol. 77, no. 4, pp. 1141-1155, 2014.

[17] J. Huang and A. C. J. Luo, "Analytical periodic motions and bifurcations in a nonlinear rotor system," International Journal of Dynamics and Control, vol. 2, no. 3, pp. 425-459, 2014.

[18] H. Hetzler, "On the effect of nonsmooth Coulomb friction on Hopf bifurcations in a $1-D o F$ oscillator with self-excitation due to negative damping," Nonlinear Dynamics, vol. 69, no. 1-2, pp. 601-614, 2012.

[19] H. Zhang, N. Zhang, F. Min, W. Yan, and E. Wang, "Bifurcations and chaos of a vibration isolation system with magnetorheological damper," AIP Advances, vol. 6, no. 3, Article ID 035310, 2016.

[20] U. Andreaus and P. Casini, "Dynamics of SDOF oscillators with hysteretic motion-limiting stop," Nonlinear Dynamics, vol. 22, no. 2, pp. 145-164, 2000.

[21] U. Andreaus and P. Casini, "Forced motion of friction oscillators limited by a rigid or deformable obstacle," Mechanics of Structures and Machines, vol. 29, no. 2, pp. 177-198, 2001.

[22] U. Andreaus and M. De Angelis, "Nonlinear dynamic response of a base-excited SDOF oscillator with double-side unilateral constraints," Nonlinear Dynamics, vol. 84, no. 3, pp. 1447-1467, 2016.

[23] G. Revel, D. M. Alonso, and J. L. Moiola, "Numerical semiglobal analysis of a 1:2 resonant Hopf-Hopf bifurcation," Physica D: Nonlinear Phenomena, vol. 247, no. 1, pp. 40-53, 2013.

[24] J. H. Yao, "O(2)-Hopf bifurcation for a model of cellular shock instability," Physica D: Nonlinear Phenomena, vol. 269, pp. 6375, 2014.

[25] Y. Huang, J. Na, X. Wu, X. Liu, and Y. Guo, "Adaptive control of nonlinear uncertain active suspension systems with prescribed performance," ISA Transactions, vol. 54, pp. 145-155, 2015.

[26] L. T. Danh and K. K. Ahn, "Active pneumatic vibration isolation system using negative stiffness structures for a vehicle seat," Journal of Sound and Vibration, vol. 333, no. 5, pp. 1245-1268, 2014.

[27] M. Silveira, B. R. Pontes Jr., and J. M. Balthazar, "Use of nonlinear asymmetrical shock absorber to improve comfort on passenger vehicles," Journal of Sound and Vibration, vol. 333, no. 7, pp. 2114-2129, 2014.

[28] Y. Kong, D. Zhao, B. Yang, C. Han, and K. Han, "Robust non-fragile $H_{\infty} / L_{2}-L_{\infty}$ control of uncertain linear system with time-delay and application to vehicle active suspension," International Journal of Robust and Nonlinear Control, vol. 25, no. 13, pp. 2122-2141, 2015.
[29] Y. Zhu and S. Zhu, "Nonlinear time-delay suspension adaptive neural network active control," Abstract and Applied Analysis, vol. 2014, Article ID 765871, 6 pages, 2014.

[30] I. I. M. Yazid, S. A. Mazlan, T. Kikuchi, H. Zamzuri, and F. Imaduddin, "Design of magnetorheological damper with a combination of shear and squeeze modes," Materials and Design, vol. 54, pp. 87-95, 2014. 


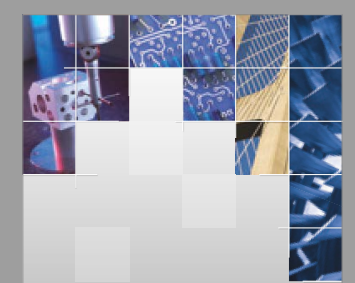

\section{Enfincering}
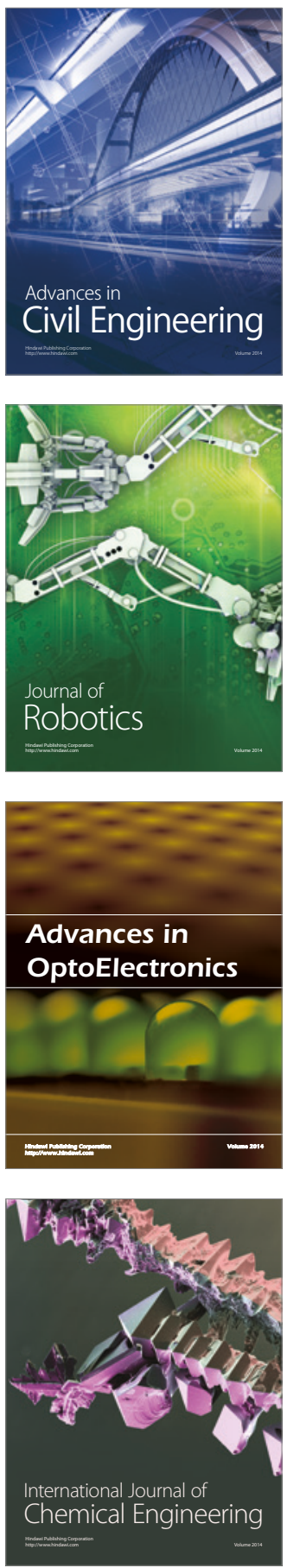

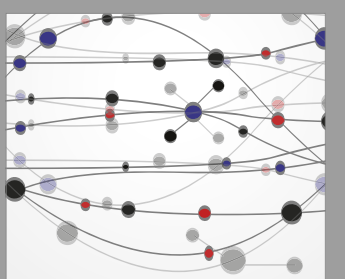

The Scientific World Journal



\section{Hindawi}

Submit your manuscripts at

https://www.hindawi.com
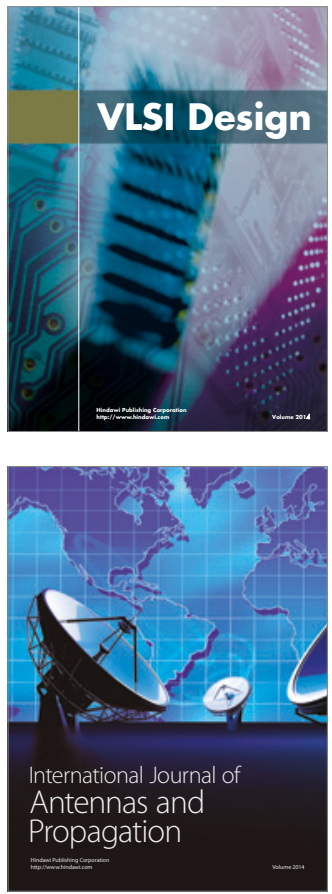

\section{Rotating}

Machinery

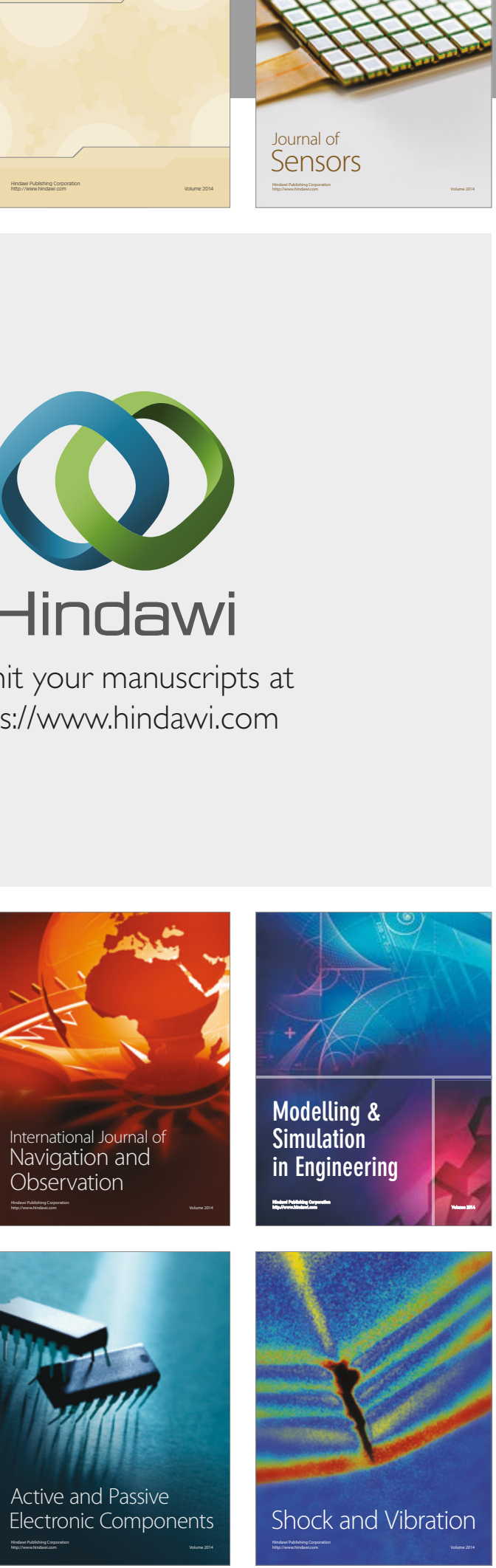


Distributed

Journal of

Control Science

and Engineering


Sensor Networks 